Document downloaded from:

http://hdl.handle.net/10251/79717

This paper must be cited as:

DANIEL CARANDO; Sevilla Peris, P. (2014). Extendibility of bilinear forms on banach sequence spaces. Israel Journal of Mathematics. 199(2):941-954. doi:10.1007/s11856-0140003-9.

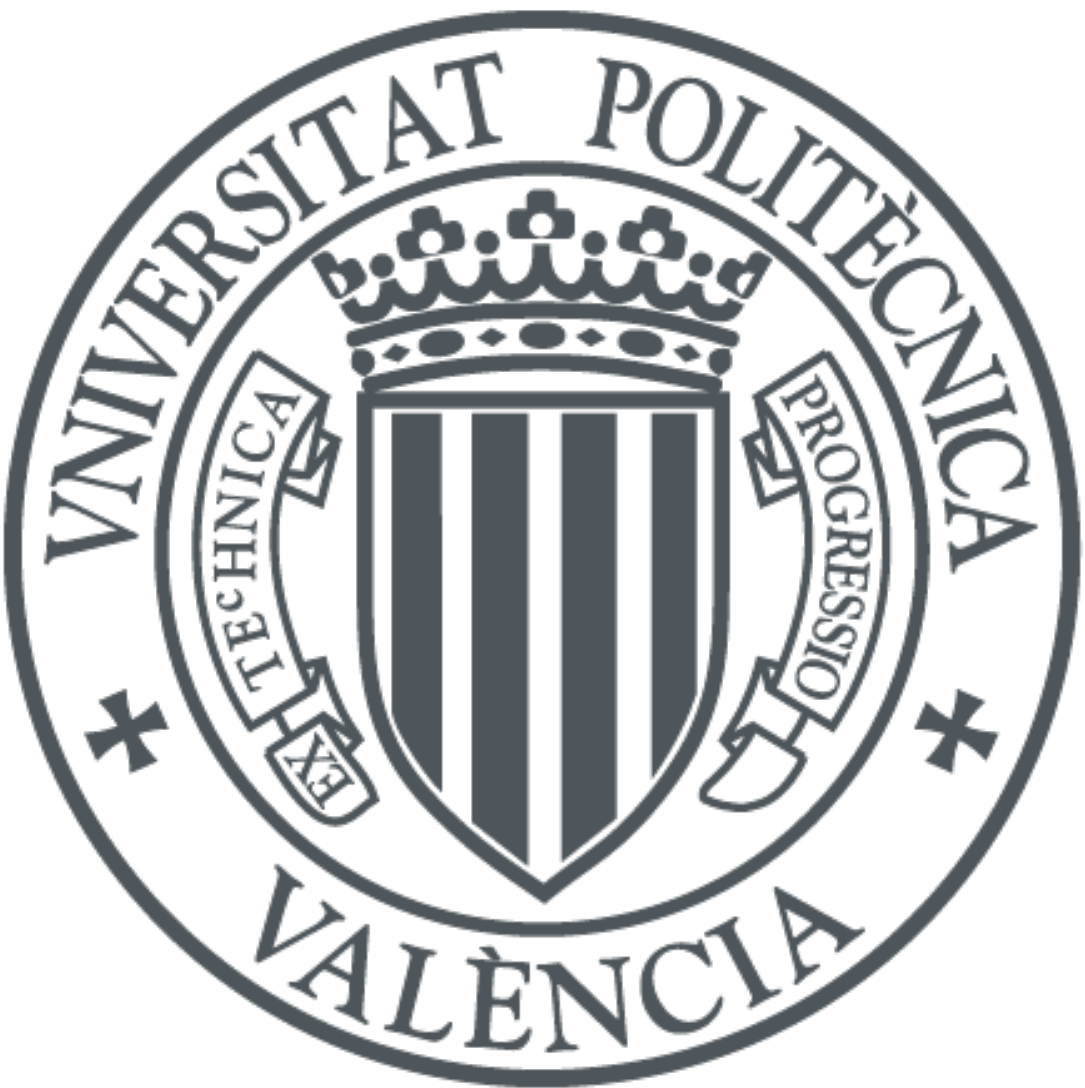

The final publication is available at

http://dx.doi.org/10.1007/s11856-014-0003-9

Copyright Springer Verlag (Germany)

Additional Information 


\title{
EXTENDIBILITY OF BILINEAR FORMS ON BANACH SEQUENCE SPACES
}

\author{
DANIEL CARANDO AND PABLO SEVILLA-PERIS
}

\begin{abstract}
We study Hahn-Banach extensions of multilinear forms defined on Banach sequence spaces. We characterize $c_{0}$ in terms of extension of bilinear forms, and describe the Banach sequence spaces in which every bilinear form admits extensions to any superspace.
\end{abstract}

\section{INTRODUCTION}

One of the fundamental results in Functional Analysis is the Hahn-Banach theorem. It was proved independently by Hahn in 1927 [18] and by Banach in 1929 [5] (see also [6, Chapitre IV, §2]). In one of its forms, it states that if $X$ is subspace of a normed space $Z$, then every continuous, linear functional $f: X \rightarrow \mathbb{K}$ can be extended to $Z$ preserving the norm. It soon became clear that a multilinear version of this result was not possible in general, and this started the search of situations on which such multilinear extension theorems are possible. A particular positive result was given by Arens in 1951, where he showed how to extend the product on a Banach algebra to its bidual and, also, how to extend bilinear operators defined on a couple of Banach spaces to their corresponding biduals $[2,3]$. This is one of the lines to find extension theorems: given a space, find a superspace to which every multilinear mapping can be extended. Aron and Berner went further on this line and showed in 1978 that every holomorphic function on a Banach space can be extended to an open subset of the bidual [4].

Another line is to fix a Banach space $X$ and consider the problem of extending bilinear forms defined on subspaces of $X$. Maurey's extension theorem [15, Corollary 12.23] is classical example of this natural point of view, in which relevant advances have been obtained in the last years $[10,26]$.

A third way to face the extension problem is to find the bilinear mappings (on a fixed Banach space) that can be extended to every superspace. This was the point of view taken by Grothendieck: in 1956 he showed in his théorème fondamental [17, page 60] that these are precisely those bilinear mappings factoring through Hilbert spaces via 2-summing operators. We say that a bilinear form $T: X \times Y \rightarrow \mathbb{K}$ is extendible (see e.g. [7, 11, 20, 22]) if for all Banach spaces $E \supset X, F \supset Y$, there exists a bilinear form defined on $E \times F$ that extends $T$. Our aim, which can be framed in this last approach, is to describe those spaces which enjoy a bilinear (or multilinear) Hahn-Banach theorem, in the sense that every bilinear form is extendible. Examples of such spaces are $A(\mathbb{D}), H^{\infty}(\mathbb{D}), \mathscr{L}^{\infty}$-spaces and Pisier spaces, but a complete characterization is still unknown. In this line, our main result is the following theroem, which solves the problem among Banach spaces with unconditional basis.

Theorem 1.1. The only Banach space with an unconditional basis on which every bilinear form is extendible is $c_{0}$.

The first author was partially supported by UBACyT W746 and CONICET PIP 0624.

The second author was supported by MICINN and FEDER Project MTM2011-22417 . 
This theorem will follow as a consequence of Theorem 2.2 below. We also characterize the Banach sequence spaces satisfying a bilinear Hahn-Banach theorem. As a byproduct, we obtain a partial answer to the following open problem: if a sequence $X_{n}$ of $n$-dimensional Banach spaces is uniformly complemented in some $\mathscr{L}_{\infty}$, must these spaces be uniformly isomorphic to $\ell_{\infty}^{n}$ ? Corollary 2.3 gives a positive answer for sections of a Banach sequence space (see Proposition 2.5).

1.1. Preliminaries. We briefly collect here some basic definitions that will be used throughout the paper. We will consider real or complex Banach spaces, that will be denoted $X$, $Y, \ldots$. Unless otherwise stated they will be assumed to be infinite dimensional. The duals will be denoted by $X^{*}, Y^{*}, \ldots$ Given two Banach spaces $X$ and $Y$, we write $X \approx Y$ if they are isomorphic and $X \underset{\approx}{\approx} Y$ if they are isometrically isomorphic. We refer to [1, 25] for basic concepts and notations on Banach spaces.

We denote $\mathscr{L}^{2}(X, Y)$ for the Banach space of all scalar valued, continuous, bilinear mappings (in short bilinear forms) on $X \times Y$. We write $\mathscr{L}^{2}(X)$ whenever $Y=X$.

The space of extendible bilinear forms is denoted by $\mathscr{E}^{2}(X, Y)$. The extendible norm

$$
\begin{array}{r}
\|T\|_{\mathscr{E}}=\|T\|_{\mathscr{E}^{2}(X, Y)}:=\inf \{c>0: \text { for all } W \supseteq X, Z \supseteq Y \text { there is an extension of } T \\
\text { to } W \times Z \text { with norm } \leq c\}
\end{array}
$$

makes $\mathscr{E}^{2}(X, Y)$ a Banach space. Since every $\ell_{\infty}(I)$ space is injective (in fact, has the metric extension property), every bilinear form on such spaces is extendible and the extendible and uniform norm coincide. Moreover, a bilinear form $T$ on $X \times Y$ is extendible if and only if it extends to $\ell_{\infty}(I) \times \ell_{\infty}(J)$, for some $\ell_{\infty}(I) \supset X$ and $\ell_{\infty}(J) \supset Y$. The supremum defining the extendible norm can be taken only over the extensions to $\ell_{\infty}(I) \times \ell_{\infty}(J)$.

We write $\mathscr{L}(X ; Y)$ for the space of all (continuous, linear) operators $u: X \rightarrow Y$. We denote $\Pi_{1}(X ; Y)$ for the space of absolutely summing operators, $\Gamma_{\infty}(X ; Y)$ for the $\infty$-factorable and $\Delta_{2}(X ; Y)$ for the 2-dominated (see $[13,15]$ for definitions and basic properties).

Given a family $\left\{X_{n}\right\}_{n}$ of Banach spaces where $\operatorname{dim} X_{n}=n$, we say that $X_{n}$ are $K$-uniformly complemented in $X$ if for each $n$ we have a mapping $i_{n}: X_{n} \rightarrow X$ and $q_{n}: X \rightarrow X_{n}$ such that $q_{n} \circ i_{n}$ is the identity on $X_{n}$ and $\left\|i_{n}\right\|\left\|q_{n}\right\| \leq K$. In this case we also say that $X$ contains $X_{n}$ uniformly complemented. We note that if $X$ contains uniform copies of $\ell_{\infty}^{n}$ (i.e., $\mathbb{K}^{n}$ with the sup norm), then the $\ell_{\infty}^{n}$ are uniformly complemented since they are injective spaces.

We are going to use the theory tensor products and operator ideals as presented in [13]. We denote $\pi$ for the projective tensor norm and $\pi \backslash$ for its right-injective associated (the largest right-injective tensor norm) and $/ \pi \backslash$ the injective associate (the largest injective tensor norm). We refer to [13] for all the basic (and not so basic) facts.

1.2. Banach sequence spaces. By a Banach sequence space (also known as Köthe sequence space) we will mean a Banach space $X \subseteq \mathbb{K}^{\mathbb{N}}$ of sequences in $\mathbb{K}$ such that $\ell_{1} \subseteq X \subseteq \ell_{\infty}$ with norm one inclusions satisfying that if $x \in \mathbb{K}^{\mathbb{N}}$ and $y \in X$ are such that $\left|x_{n}\right| \leq\left|y_{n}\right|$ for all $n \in \mathbb{N}$, then $x$ belongs to $X$ and $\|x\| \leq\|y\|$.

If $X$ is a Banach sequence space, we denote by $\left\{e_{n}\right\}_{n}$ the sequence of canonical vectors, which is always a 1-unconditional basic sequence. We define $X_{N}=\operatorname{span}\left\{e_{1}, \ldots, e_{N}\right\}$ and $X_{0}=\overline{\operatorname{span}}\left\{e_{n}\right\}_{n}$. This last space is usually referred to as the minimal kernel of $X$. Given $x \in X$ we write $x^{N}=\left(x_{1}, \ldots, x_{N}\right)$. There are inclusions $i_{N}^{X}: X_{N} \hookrightarrow X$ and projections $\pi_{N}^{X}: X \rightarrow X_{N}$ given by $i_{N}^{X}\left(x_{1}, \ldots, x_{N}\right)=\left(x_{1}, \ldots, x_{N}, 0,0, \ldots\right)$ and $\pi_{N}^{X}(x)=x^{N}$. The 
inclusions are isometric and the projections have norm 1 . For the case $X=\ell_{p}(1 \leq p \leq \infty)$, we write $\ell_{p}^{N}$ for $X_{N}$.

Given a Banach sequence space $X$, its Köthe dual is defined as

$$
X^{\times}=\left\{\left(z_{n}\right)_{n} \in \mathbb{K}^{\mathbb{N}}: \sum_{n}\left|z_{n} x_{n}\right|<\infty \text { for all } x \in X\right\} .
$$

With the norm $\|z\|_{X^{\times}}=\sup _{\|x\|_{X} \leq 1} \sum_{n}\left|z_{n} x_{n}\right|$ it is again a Banach sequence space.

Following [24, 1.d], a Banach sequence space $X$ is said to be $r$-convex (with $1 \leq r<\infty$ ) if there exists a constant $\kappa>0$ such that for any choice $x_{1}, \ldots, x_{N} \in X$ we have

$$
\left\|\left(\left(\sum_{j=1}^{N}\left|x_{j}(k)\right|^{r}\right)^{1 / r}\right)_{k=1}^{\infty}\right\|_{X} \leq \kappa\left(\sum_{j=1}^{N}\left\|x_{j}\right\|_{X}^{r}\right)^{1 / r} .
$$

On the other hand, $X$ is $s$-concave (with $1 \leq s<\infty$ ) if there is a constant $\kappa>0$ such that

$$
\left(\sum_{j=1}^{N}\left\|x_{j}\right\|_{X}^{s}\right)^{1 / s} \leq \kappa\left\|\left(\left(\sum_{j=1}^{N}\left|x_{j}(k)\right|^{s}\right)^{1 / s}\right)_{k=1}^{\infty}\right\|_{X}
$$

for all $x_{1}, \ldots, x_{N} \in X$.

It is well known that $\ell_{p}$ is $r$ convex for $1 \leq r \leq p$ and $s$-concave for $p \leq s<\infty$.

The following result is probably known. However we were not able to find a proper reference of this fact and we include here a short proof. It is modelled along the same lines as the proof of the fact that if the canonical vectors form a basis of $X$ then both duals coincide.

Proposition 1.2. If $X$ is a Banach sequence space, its Köthe dual $X^{\times}$is a 1-complemented subspace of the usual dual $X^{*}$.

Proof. Let us see first that the mapping $i: X^{\times} \rightarrow X^{*}$ defined by $i(z)=\varphi_{z}: X \rightarrow \mathbb{K}$, with $\varphi_{z}(x)=\sum_{n} z_{n} x_{n}$, is an isometry. It is clearly well defined; moreover

$$
\left\|\varphi_{z}\right\|=\sup _{x \in B_{X}}\left|\sum_{n} z_{n} x_{n}\right| \leq \sup _{x \in B_{X}} \sum_{n}\left|z_{n} x_{n}\right|=\|z\|_{X^{\times}} .
$$

To see the reverse inequality, for any $t, s \in \mathbb{K}$ we take $\varepsilon(t, s) \in \mathbb{K}$ with $|\varepsilon(t, s)|=1$ such that $|s t|=\varepsilon(t, s) s t$; then for every $x \in B_{X}$ and every $z \in X^{\times}$we have

$$
\sum_{n}\left|z_{n} x_{n}\right|=\sum_{n} \varepsilon\left(z_{n}, x_{n}\right) z_{n} x_{n}=\left|\sum_{n} \varepsilon\left(z_{n}, x_{n}\right) z_{n} x_{n}\right| \leq \sup _{a \in B_{X}}\left|\sum_{n} z_{n} a_{n}\right|=\left\|\varphi_{z}\right\|,
$$

which gives $\|z\|_{X^{\times}} \leq\left\|\varphi_{z}\right\|$.

On the other hand, the mapping $q: X^{*} \rightarrow X^{\times}$given by $q(\varphi)=\left(\varphi\left(e_{n}\right)\right)_{n}$ defines a norm-one projection. Indeed, given $x \in X$ and fixed $N$ we have

$$
\begin{aligned}
\sum_{n=1}^{N}\left|x_{n} \varphi\left(e_{n}\right)\right|=\sum_{n=1}^{N} \varepsilon\left(x_{n}, \varphi\left(e_{n}\right)\right) x_{n} \varphi\left(e_{n}\right)= & \varphi\left(\sum_{n=1}^{N} \varepsilon\left(x_{n}, \varphi\left(e_{n}\right)\right) x_{n} e_{n}\right) \\
& \leq\|\varphi\|\left\|\sum_{n=1}^{N} \varepsilon\left(x_{n}, \varphi\left(e_{n}\right)\right) x_{n} e_{n}\right\|_{X} \leq\|\varphi\|\|x\| .
\end{aligned}
$$

This shows that $\sum_{n=1}^{\infty}\left|x_{n} \varphi\left(e_{n}\right)\right| \leq\|\varphi\|\|x\|$, which gives that $q$ is well defined and $\|q(\varphi)\| \leq$ $\|\varphi\|$. Furthermore, $q$ is a projection, since clearly $q \circ i(z)=q\left(\varphi_{z}\right)=\left(z_{n}\right)_{n}=z$. 


\section{Extension of Bilinear forms on BANACH SEQUence spaCeS}

In what follows $K_{G}$ denotes the Grothendieck's constant. We begin by proving the following known fact, which was stated as Theorem 3.4 in [20] without the estimates for the norms (see [11, Lemma 2.4] for a result in the same spirit).

Proposition 2.1. If every bilinear form $B: X \times Y \rightarrow \mathbb{K}$ is extendible with $\|B\|_{\mathscr{E}} \leq K\|B\|$, then every operator $u: X^{*} \rightarrow \ell_{2}$ is absolutely 1 -summing and $\pi_{1}(u) \leq K_{G} K\|u\|$.

Proof. We first note that, by definition of the tensor norm $/ \pi \backslash$ (see [13, Section 20.7]), $\mathscr{E}^{2}(X, Y)$ is isometrically the dual of $X \otimes / \pi \backslash Y$. Then, our hypothesis is equivalent to the inequality $\pi \leq K / \pi \backslash$ on $X \otimes Y$ and, as a consequence, we also have $\pi \backslash \leq K / \pi \backslash$ on $X \otimes Y$. Since both $\pi \backslash$ and $/ \pi \backslash$ are right-injective, an application of Dvoretzky's theorem [15, 19.1] and the previous inequality gives an isomorphism

$$
X \otimes_{/ \pi \backslash} \ell_{2}^{N} \longrightarrow X \otimes_{\pi \backslash} \ell_{2}^{N}
$$

with norm at most $K$. Since $\ell_{2}^{N}$ is finite dimensional, $\mathscr{L}\left(X ; \ell_{2}^{N}\right)$ and $X^{*} \otimes \ell_{2}^{N}$ coincide as sets. Then, the embedding in [13, Section 17.6] is actually surjective and [13, Sections 21.5 and 27.2] give $X^{*} \otimes_{w_{\infty}} \ell_{2}^{N} \stackrel{1}{\approx} \Gamma_{\infty}\left(X ; \ell_{2}^{N}\right)$ (see [15, Chapter 9] or [13, Section 18] for the definition of $\left.\Gamma_{\infty}(X ; Y)\right)$. Therefore,

$$
\left(X \otimes_{\pi \backslash} \ell_{2}^{N}\right)^{* *} \underset{\sim}{\approx}\left(\Gamma_{\infty}\left(X, \ell_{2}^{N}\right)\right)^{*} \stackrel{1}{\approx}\left(X^{*} \otimes_{w_{\infty}} \ell_{2}^{N}\right)^{*} \underset{\sim}{\approx} \Pi_{1}\left(X^{*} ; \ell_{2}^{N}\right) .
$$

Now, by [13, Sections 17.12 and 27.2], the operator ideal $\Delta_{2}$ is associated to $w_{2}$ and $\Pi_{1}$ is associated to $\pi \backslash$. On the other hand Grothendieck's inequality [13, Section 20.17] states that $w_{2} \geq K_{G} / \pi \backslash$ and clearly we have $\mathscr{L}\left(X^{*} ; \ell_{2}^{N}\right) \stackrel{1}{\approx} \Delta_{2}\left(X^{*} ; \ell_{2}^{N}\right)$. Using (2) to take biduals in (1) we have an isomorphism

$$
\mathscr{L}\left(X^{*} ; \ell_{2}^{N}\right) \longrightarrow\left(X \otimes_{/ \pi \backslash} \ell_{2}^{N}\right)^{* *} \longrightarrow\left(X^{* *} \otimes_{\pi \backslash} \ell_{2}^{N}\right)^{* *} \stackrel{1}{\approx} \Pi_{1}\left(X^{*} ; \ell_{2}^{N}\right),
$$

where the first mapping has norm bounded by $K_{G}$ and the second one by $K$. Since both $\mathscr{L}$ and $\Pi_{1}$ are maximal operator ideals, the same holds if we put $\ell_{2}$ instead of $\ell_{2}^{N}$.

With this result we can now prove the following one, from which Theorem 1.1 follows as an immediate consequence.

Theorem 2.2. Let $X$ be a Banach space with an unconditional basis and $Y$ be any infinite dimensional Banach space such that every bilinear form on $X \times Y$ is extendible. Then $X \approx c_{0}$.

Proof. Let us see first that, under our assumptions, the basis of $X$ must be shrinking. Suppose it is not. Since it is unconditional, by James theorem [19, Corollary 2] (see also [1, Theorem 3.3.1]) $X$ must contain a complemented copy of $\ell_{1}$. Since the property of all bilinear forms being extendible is inherited by complemented subspaces, it follows that every bilinear form on $\ell_{1} \times Y$ is extendible. This implies [22, Lemma 6] that every continuous linear operator from $Y$ to $\ell_{\infty}$ is absolutely 2 -summing. By the so called $\mathfrak{L}_{p}$-Local Technique Lemma for Operator Ideals [13, Section 23.1], the same holds for every operator from $Y$ to $\ell_{\infty}(I)$, for any index set $I$. But this is not possible, since there exists an isometric embedding from $Y$ into some $\ell_{\infty}(I)$, and this cannot be absolutely 2-summing (otherwise, the identity on $Y$ would be so, but $Y$ is infinite dimensional).

This means that the canonical basis of $X$ must be shrinking. We can assume that the basis is 1-unconditional, so that the coordinate basis is an 1-unconditional basis of $X^{*}$. We also 
know from Proposition 2.1 that all operators from $X^{*}$ to $\ell_{2}$ are absolutely 1 -summing. By [23] (see also [25, Theorem 8.21]) this implies that the basis of $X^{*}$ is $\left(K_{G} K\right)^{2}$-equivalent to the basis of $\ell_{1}$. Although $\ell_{1}$ has many non-isomorphic preduals, if the coordinate basis is equivalent to that of $\ell_{1}$, a standard computation shows that the canonical basis on $X$ must be $\left(K_{G} K\right)^{2}$-equivalent to the basis of $c_{0}$.

Note that the proof not only shows that $X$ must be isomorphic to $c_{0}$, but also gives an estimation of the Banach-Mazur distance between $X$ and $c_{0}$ whenever $X$ has an 1-unconditional basis. As a consequence, we can also characterize the pairs of Banach sequence spaces on which every bilinear form is extendible.

Corollary 2.3. If $X$ and $Y$ are Banach sequence spaces, then the following are equivalent.

(i) $\mathscr{L}^{2}(X, Y)=\mathscr{E}^{2}(X, Y)$ and $\|B\|_{\mathscr{E}} \leq K_{1}\|B\|$ for all bilinear form $B$ on $X \times Y$.

(ii) The canonical basic sequence of $X$ and $Y$ are $K_{2}$-equivalent to the canonical basis of $c_{0}$.

(iii) $K_{3}:=\sup \left\{d\left(X_{N}, \ell_{\infty}^{N}\right), d\left(Y_{N}, \ell_{\infty}^{N}\right): N \in \mathbb{N}\right\}$ is finite (where d denotes the BanachMazur distance).

(iv) The spaces $X_{N}$ and $Y_{N}(N \in \mathbb{N})$ are $K_{4}$-uniformly complemented in some $L_{\infty}(\mu)$.

Moreover, we have $K_{4} \leq K_{3} \leq K_{2} \leq\left(K_{G} K_{1}\right)^{2}$ and $K_{1} \leq K_{2}^{2} \leq K_{G}^{4} K_{4}^{8}$.

Proof. If (i) holds on $X \times Y$, then the same holds for $X_{N} \times Y_{N}$ for any $N$ and, by the density lemma [13, Section 13.4], for $X_{0} \times Y_{0}$. By Theorem 2.2, both bases are $\left(K_{G} K\right)^{2}$-equivalent to the basis of $c_{0}$.

The implications (ii) $\Rightarrow$ (iii) $\Rightarrow$ (iv) are immediate, as well as the inequalities $K_{4} \leq K_{3} \leq$ $K_{2}$.

If (iv) holds, bilinear forms on $X_{N} \times Y_{N}$ are extendible with $\|\cdot\|_{\mathscr{E}} \leq K_{4}^{2}\|\cdot\|$ and, as before, the same holds for $X_{0} \times Y_{0}$. By Theorem 2.2 their canonical bases are $K_{G}^{2} K_{4}^{4}$-equivalent to the canonical basis of $c_{0}$, which is (ii).

Now suppose (ii) holds and take a bilinear form $B: X \times Y \rightarrow \mathbb{K}$. We know from Proposition 1.2 that $X^{\times}$is 1-complemented in $X^{*}$. Then $\left(X^{\times}\right)^{*}$ is isometrically a (complemented) subspace of $X^{* *}$. Since (ii) implies $X^{\times}=\ell_{1}$, we also have $\left(X^{\times}\right)^{*}=X^{\times \times}=\ell_{\infty}$. The same holds for $Y$, so we obtain the following diagrams:
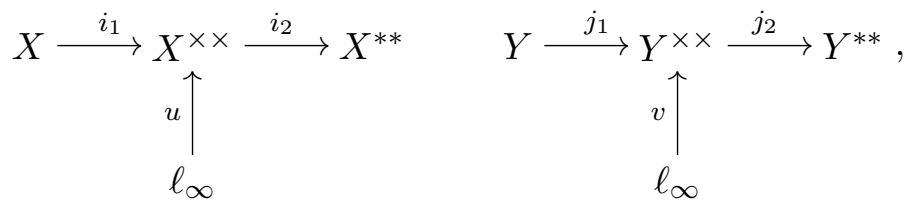

where $i_{1}, i_{2}, j_{1}$ and $j_{2}$ are isometric injections and $u$ and $v$ are isomorphisms with

$$
\|u\|\left\|u^{-1}\right\| \leq K_{2} \quad \text { and }\|v\|\left\|v^{-1}\right\| \leq K_{2} .
$$

We can extend $B$ (in the canonical way) to a bilinear form $\tilde{B}: X^{* *} \times Y^{* *} \rightarrow \mathbb{K}$ with the same norm as $B$, and then define a bilinear form $\widehat{B}$ on $\ell_{\infty} \times \ell_{\infty}$ by $\widehat{B}=\tilde{B} \circ\left(i_{2} \circ u, j_{2} \circ v\right)$. We have obtained the factorization $B=\widehat{B} \circ\left(u^{-1} \circ i_{1}, v^{-1} \circ j_{1}\right)$. Since on $\ell_{\infty} \times \ell_{\infty}$ every bilinear form is extendible (with the extendible norm equal to the usual norm), from the ideal property of extendible bilinear forms and inequalities (4) we conclude that $B$ is extendible and $\|B\|_{\mathscr{E}} \leq K_{2}^{2}\|B\|$. 
It follows from the previous corollary (and its proof) that a Banach sequence space on which every bilinear form is extendible must satisfy the sublattice inclusions

$$
c_{0} \subset X \subset \ell_{\infty} .
$$

Conversely, let $X$ be a Banach sequence space satisfying (5). By a closed graph argument both inclusions are continuous and it is easy to check that $X$ satisfies the equivalent conditions of Corollary 2.3. As a consequence, we have the following version of Theorem 1.1 for Banach sequence spaces.

Corollary 2.4. The Banach sequence spaces $X$ on which every bilinear form is extendible are those satisfying (5). This happens if and only if $X^{\times}=\ell_{1}$.

Examples of such spaces are $c_{0} \oplus \ell_{\infty}, c_{0}\left(\ell_{\infty}\right)$ and $\ell_{\infty}\left(c_{0}\right)$. It is not hard to see that these spaces are mutually non-isomorphic Banach sequence spaces (see also [12], where the authors show that $c_{0}\left(\ell_{\infty}\right)$ and $\ell_{\infty}\left(c_{0}\right)$ are not isomorphic even as Banach spaces).

If a sequence $X_{n}$ of $n$-dimensional Banach spaces is uniformly complemented in some $\mathscr{L}_{\infty}$, it is an open problem if these spaces have to be uniformly isomorphic to $\ell_{\infty}^{n}$. Taking $X=Y$ in Corollary 2.3, the implication (iv) $\Rightarrow$ (iii) gives the following partial answer.

Proposition 2.5. If the $N$-dimensional sections $X_{N}$ of a Banach sequence space $X$ are uniformly complemented in some $L_{\infty}(\mu)$, then they must be uniformly isomorphic to $\ell_{\infty}^{N}$.

Note also that, since $\ell_{\infty}$ and $c_{0}$ are the only symmetric Banach sequence spaces satisfying (ii) of Corollary 2.3, these two are the only symmetric Banach sequence spaces on which every bilinear form is extendible.

If $X_{1}, \ldots, X_{n}$ are Banach spaces such that every $n$-linear form on $X_{1} \times \cdots \times X_{n}$ is extendible, then it is known (and easy to see) that so is every bilinear form on $X_{i} \times X_{j}$ for each pair $i \neq j$. Indeed, given $B \in \mathscr{L}^{2}\left(X_{i} \times X_{j}\right)$, we can multiply it by linear functionals to obtain a $n$-linear form on $X_{1} \times \cdots \times X_{n}$. This is extendible by our hypothesis. From this, it is rather immediate to conclude that $B$ is extendible. As a consequence, multilinear versions of our results follow directly from the bilinear ones.

If $X$ and $Y$ are Banach sequence spaces such that every bilinear form on $X \times Y$ is extendible, then we know from Theorem 2.3 (iii) that both $X$ and $Y$ contain the $\ell_{\infty}^{N}$ uniformly. We can extend this statement to subspaces of Banach lattices.

Proposition 2.6. Let $X_{1}, X_{2}$ be subspaces of Banach lattices such that every $n$-linear form on $X_{1}, X_{2}$ is extendible. Then every infinite dimensional complemented subspace of each $X_{j}$ contains the $\ell_{\infty}^{N}$ uniformly.

Proof. Suppose that there exists a complemented subspace $E$ of $X_{1}$ that does not contain the $\ell_{\infty}^{N}$ uniformly. By [21, Corollary 1], $E$ must contain uniformly complemented $N$-dimensional subspaces $E_{N}$ such that $\sup _{N} d\left(E_{N}, \ell_{p}^{N}\right)<\infty$ for $p=1$ or 2 . Since $E$ is complemented in $X_{1}$, the $E_{N}$ are also uniformly complemented in $X_{1}$. On the other hand, again by [21, Corollary 1], $X_{2}$ must contain uniformly complemented $N$-dimensional subspaces $F_{N}$ such that $\sup _{N} d\left(F_{N}, \ell_{q}^{N}\right)<\infty$ for $q=1,2$ or $\infty$. Our hypotheses ensure that bilinear forms on $X_{1} \times X_{2}$ are extendible. Since $E_{N}$ and $F_{N}$ are uniformly complemented in $X_{1}$ and $X_{2}$ and they are (uniformly) isomorphic to $\ell_{p}^{N}$ and $\ell_{q}^{N}$, there must exist $K>0$ such that $\|B\|_{\mathscr{E}^{2}\left(\ell_{p}^{N}, \ell_{q}^{N}\right)} \leq$ $K\|B\|_{\mathscr{L}^{2}\left(\ell_{p}^{N}, \ell_{q}^{N}\right)}$ for all $N$. Now, the density lemma [13, Section 13.4] implies that every bilinear form on $\ell_{p} \times \ell_{q}\left(\right.$ or $\left.\ell_{p} \times c_{0}\right)$ must be extendible, which contradicts Theorem 2.2. 
The converse of Proposition 2.6 does not hold. For example, the Schreier space is $c_{0^{-}}$ saturated and there are non-extendible bilinear forms on it (since there are bilinear forms which are not weakly sequentially continuous). Another conterexample of the converse is $d_{*}(w, 1)$, the predual of the Lorentz sequence space $d(w, 1)$ (see [27] or [16] for a description of the predual). Since these examples are Banach sequence spaces, they also show that assertion (iii) in Theorem 2.3 is strictly stronger than containing $\ell_{\infty}^{N}$ uniformly.

In Banach sequence spaces, diagonal bilinear forms are the simplest ones. These are the bilinear forms $T_{\alpha}: X_{1} \times X_{2} \rightarrow \mathbb{C}$ given by

$$
T_{\alpha}\left(x^{1}, x^{2}\right)=\sum_{k=1}^{\infty} \alpha_{k} x_{k}^{1} x_{k}^{2},
$$

for some sequence $\left(\alpha_{k}\right)_{k}$ of scalars. We end this note showing, under some assumptions, which are the spaces on which all diagonal bilinear forms are extendible.

Following standard notation, given a symmetric Banach sequence space $X$ we consider the fundamental function of $X$, given by $\lambda_{X}(N):=\left\|\sum_{k=1}^{N} e_{k}\right\|_{X}$ for $N \in \mathbb{N}$.

Given two sequence of real numbers $\left(a_{n}\right)_{n}$ and $\left(b_{n}\right)_{n}$ we write $a_{n} \preceq b_{n}$ whenever there is a universal constant $C>0$ such that $a_{n} \leq C b_{n}$ for every $n$. If $a_{n} \preceq b_{n}$ and $b_{n} \preceq a_{n}$, we write $a_{n} \asymp b_{n}$.

Theorem 2.7. Let $X$ and $Y$ be symmetric Banach sequence spaces, each being 2-convex or 2-concave. Then all diagonal bilinear forms on $X \times Y$ are extendible if and only if either $X=Y=\ell_{1}$ or $X, Y \in\left\{c_{0}, \ell_{\infty}\right\}$

Proof. The if part is clear: by [8, Proposition 2.3] (see also [9, Proposition 1.2]) on $\ell_{1}$ diagonal bilinear forms are integral (and, therefore, extendible), and in the other cases all bilinear forms are extendible.

For the converse, we consider the diagonal bilinear form given by $\phi_{N}(x, y)=\sum_{i=1}^{N} x_{i} y_{i}$. It is easily computed that $\left\|\phi_{N}\right\|_{\mathscr{L}^{2}\left(\ell_{2}^{N}\right)}=1$; on the other hand, by [8, Proposition 1.1] or $\left[11\right.$, Proposition 2.5] we have $\left\|\phi_{N}\right\|_{\mathscr{E}^{2}\left(\ell_{2}^{N}\right)}=\left\|\phi_{N}\right\|_{\mathscr{N}^{2}\left(\ell_{2}^{N}\right)}=N$. Let now $\operatorname{id}_{X}^{N}: \ell_{2}^{N} \rightarrow X_{N}$ and $\operatorname{id}_{Y}^{N}: \ell_{2}^{N} \rightarrow Y_{N}$ be the identity mappings. Comparing the usual and extendible norms of the bilinear forms $\phi_{N}$ and $\phi_{N} \circ\left(\left(\operatorname{id}_{X}^{N}\right)^{-1},\left(\operatorname{id}_{Y}^{N}\right)^{-1}\right)$, we get

$$
N \preceq\left\|\operatorname{id}_{X}^{N}\right\|\left\|\operatorname{id}_{Y}^{N}\right\|\left\|\left(\operatorname{id}_{X}^{N}\right)^{-1}\right\|\left(\operatorname{id}_{Y}^{N}\right)^{-1} \| .
$$

By $[28,16.4]$ (see also [14, page 138]), since $X$ is a symmetric Banach sequence space we have $d\left(\ell_{2}^{N}, X_{N}\right)=\left\|\operatorname{id}_{X}^{N}\right\|\left\|\left(\operatorname{id}_{X}^{N}\right)^{-1}\right\|$ (and the same for $Y_{N}$ ). Therefore,

$$
N \preceq\left\|\operatorname{id}_{X}^{N}\right\|\left\|\operatorname{id}_{Y}^{N}\right\|\left\|\left(\operatorname{id}_{X}^{N}\right)^{-1}\right\|\left(\operatorname{id}_{Y}^{N}\right)^{-1} \|=d\left(\ell_{2}^{N}, X_{N}\right) d\left(\ell_{2}^{N}, Y_{N}\right) .
$$

Since we always have $d\left(\ell_{2}^{N}, X_{N}\right) \leq \sqrt{N}$ and $d\left(\ell_{2}^{N}, Y_{N}\right) \leq \sqrt{N}$, we can conclude that $\sqrt{N} \asymp$ $d\left(\ell_{2}^{N}, X_{N}\right)=\left\|\operatorname{id}_{X}^{N}\right\|\left\|\left(\operatorname{id}_{X}^{N}\right)^{-1}\right\|$ (and the same for $\left.Y_{N}\right)$. We now apply [14, Lemma 1 (i)] and get:

$$
\max \left(\frac{1}{\lambda_{X}(N)}, \frac{\lambda_{X}(N)}{N}\right) \asymp 1
$$

From this we can conclude that $X$ must be $\ell_{1}, c_{0}$ or $\ell_{\infty}$. Indeed, suppose we split the natural numbers $\mathbb{N}=I \cup J$, so that $\left(\frac{1}{\lambda_{X}(N)}\right)_{N \in I} \asymp 1$ and $\left(\frac{\lambda_{X}(N)}{N}\right)_{N \in J} \asymp 1$. We have then that $\left(\lambda_{X}(N)\right)_{N \in I}$ is bounded and $(N)_{N \in J} \preceq\left(\lambda_{X}(N)\right)_{N \in J}$. Since $\left(\lambda_{X}(N)\right)_{N \in \mathbb{N}}$ is non-decreasing, either $I$ or $J$ must be finite. If $J$ is finite, then $\left(\lambda_{X}(N)\right)_{N \in \mathbb{N}}$ is bounded and then the 
norm in $X$ is equivalent to the sup norm and $X$ is $c_{0}$ or $\ell_{\infty}$. If $I$ is finite, then $N \preceq$ $\left(\lambda_{X}(N)\right)_{N \in \mathbb{N}}$. Although the fundamental sequence of a symmetric Banach sequence space does not characterize the norm, for this extreme case it is possible to prove that the norm on $X$ must be isomorphic to $\ell_{1}$ : from the estimate $N \preceq\left(\lambda_{X}(N)\right)_{N \in \mathbb{N}}$ we easily obtain $\lambda_{X \times}(N) \asymp 1$ and, by the previous case, $X^{\times}$must be $\ell_{\infty}$. Then we have $X=\ell_{1}$. Proceeding in the same way, $Y$ has to be either $\ell_{1}, c_{0}$ or $\ell_{\infty}$.

It remains to show that on $c_{0} \times \ell_{1}$ and on $\ell_{1} \times \ell_{\infty}$ there are non-extendible diagonal bilinear forms. The mapping $c_{0} \times \ell_{1}$ given by $\left(x, x^{\prime}\right) \mapsto x^{\prime}(x)$ is the diagonal bilinear form induced by the formal identity. An extension of this mapping to $c_{0} \times \ell_{\infty}$ would give a projection from $\ell_{\infty}$ to $\ell_{1}$ (see $[13,1.5]$ ), which does not exist. For $\ell_{1} \times \ell_{\infty}$ we can reason in a similar way.

Both assumptions on symmetry and concavity/convexity in "only if" part of the previous theorem cannot be omitted. Indeed, if we take $c_{0} \oplus \ell_{1}$ (that seen as a sequence space is neither symmetric nor 2-concave or 2-convex), then every diagonal bilinear form on $\left(c_{0} \oplus \ell_{1}\right) \times\left(c_{0} \oplus \ell_{1}\right)$ is the sum of a diagonal bilinear form on $c_{0} \times c_{0}$ and a diagonal bilinear form on $\ell_{1} \times \ell_{1}$, and is therefore extendible.

\section{ACKNOWLEDGEMENTS}

We also wish to thank the referees, We wish to thank Andreas Defant for valuable comments and useful conversations that improved the paper. for suggestions which improved the final shape of the paper and for pointing to us the question considered in Corollary 2.4 Proposition 2.5.

\section{REFERENCES}

[1] F. Albiac and N. J. Kalton. Topics in Banach space theory, volume 233 of Graduate Texts in Mathematics. Springer, New York, 2006.

[2] R. Arens. The adjoint of a bilinear operation. Proc. Amer. Math. Soc., 2:839-848, 1951.

[3] R. Arens. Operations induced in function classes. Monatsh. Math., 55:1-19, 1951.

[4] R. M. Aron and P. D. Berner. A Hahn-Banach extension theorem for analytic mappings. Bull. Soc. Math. France, 106(1):3-24, 1978.

[5] S. Banach. Sur les fonctionelles linéaires. Studia Math., 1(2):211-216, 1929.

[6] S. Banach. Théorie des opérations linéaires. (Monogr. Mat. 1) Warszawa: Subwncji Funduszu Narodowej. VII, 254 S., Warsaw, 1932.

[7] D. Carando. Extendible polynomials on Banach spaces. J. Math. Anal. Appl., 233(1):359-372, 1999.

[8] D. Carando. Extendibility of polynomials and analytic functions on $l_{p}$. Studia Math., 145(1):63-73, 2001.

[9] D. Carando, V. Dimant, and P. Sevilla-Peris. Limit orders and multilinear forms on $l_{p}$ spaces. Publ. Res. Inst. Math. Sci., 42(2):507-522, 2006.

[10] J. M. F. Castillo, R. García, A. Defant, D. Pérez-García, J. Suárez. Local complementation and the extension of bilinear mappings. Math. Proc. Cambridge Philos. Soc. 152 (2012), no. 1, 153166

[11] J. M. F. Castillo, R. García, and J. A. Jaramillo. Extension of bilinear forms on Banach spaces. Proc. Amer. Math. Soc., 129(12):3647-3656 , 2001.

[12] P. Cembranos, J. Mendoza. The Banach spaces $\ell_{\infty}(c 0)$ and $c_{0}\left(\ell_{\infty}\right)$ are not isomorphic. J. Math. Anal. Appl. 367 (2):461-463, 2010.

[13] A. Defant and K. Floret. Tensor norms and operator ideals, volume 176 of North-Holland Mathematics Studies. North-Holland Publishing Co., Amsterdam, 1993.

[14] A. Defant and C. Michels. Norms of tensor product identities. Note Mat., 25(1):129-166, 2005/06.

[15] J. Diestel, H. Jarchow, and A. Tonge. Absolutely summing operators, volume 43 of Cambridge Studies in Advanced Mathematics. Cambridge University Press, Cambridge, 1995.

[16] D. J. H. Garling. On symmetric sequence spaces. Proc. London Math. Soc. (3), 16:85-106, 1966.

[17] A. Grothendieck. Résumé de la théorie métrique des produits tensoriels topologiques. Bol. Soc. Mat. São Paulo, 8:1-79, 1953. 
[18] H. Hahn. über lineare Gleichungssysteme in linearen Räumen. J. Reine Angew. Math., 157:214-229, 1927.

[19] R. C. James. Bases and reflexivity of Banach spaces. Ann. of Math. (2), 52:518-527, 1950.

[20] H. Jarchow, C. Palazuelos, D. Pérez-García, and I. Villanueva. Hahn-Banach extension of multilinear forms and summability. J. Math. Anal. Appl., 336(2):1161-1177, 2007.

[21] W. B. Johnson and L. Tzafriri. On the local structure of subspaces of Banach lattices. Israel J. Math., 20(3-4):292-299, 1975.

[22] P. Kirwan and R. A. Ryan. Extendibility of homogeneous polynomials on Banach spaces. Proc. Amer. Math. Soc., 126(4):1023-1029, 1998.

[23] J. Lindenstrauss and A. Pełczyński. Absolutely summing operators in $L_{p}$-spaces and their applications. Studia Math., 29:275-326, 1968.

[24] J. Lindenstrauss and L. Tzafriri. Classical Banach spaces. II, volume 97 of Ergebnisse der Mathematik und ihrer Grenzgebiete [Results in Mathematics and Related Areas]. Springer-Verlag, Berlin, 1979. Function spaces.

[25] G. Pisier. Factorization of linear operators and geometry of Banach spaces, volume 60 of CBMS Regional Conference Series in Mathematics. Published for the Conference Board of the Mathematical Sciences, Washington, DC, 1986.

[26] M. Fernndez-Unzueta, A. Prieto. Extension of polynomials defined on subspaces. Math. Proc. Cambridge Philos. Soc. 148 (2010), no. 3, 505518.

[27] W. L. C. Sargent. Some sequence spaces related to the $l^{p}$ spaces. J. London Math. Soc., 35:161-171, 1960.

[28] N. Tomczak-Jaegermann. Banach-Mazur distances and finite-dimensional operator ideals, volume 38 of Pitman Monographs and Surveys in Pure and Applied Mathematics. Longman Scientific \& Technical, Harlow, 1989.

Departamento de Matemática - Pab I, Facultad de Cs. Exactas y Naturales, Universidad de Buenos Aires, (1428) Buenos Aires, Argentina And IMAS - CONiCET

E-mail address: dcarando@dm.uba.ar

Instituto Universitario de Matemática Pura y Aplicada and DMA, ETSiamn, Universitat Politècnica de VAlÈnCia, VAlencia, Spain

E-mail address: psevilla@mat.upv.es 